\title{
Melanins are Biological Diamonds
}

\author{
L. Venger \\ Email: luvenger94@gmail.com)
}

\begin{abstract}
In the article the physical, chemical, biological properties, the significance and the role in Nature of melanin pigments are considered. Very long time these pigments were considered as metabolism garbage in the living organisms. The comparison of the only same results and facts from the huge masses of the science information with main principles of the new science paradigm - the Theory of Torsion Fields and the Theory of Physical Vacuum - allow to change the view on the true invention, the biological role and the functions of Melanins in Nature. Many theoretical and experimental facts specify on the active participation of melanins in the energyand-informative processes and their first degree role on the all evolution stages of living organisms: from the chemical evolution to the brain and consciousness functions. In the sense Melanins are the most precious substance for the living organisms, true their Diamonds.
\end{abstract}

Keywords: living organism, melanin, pigment, torsion field, physical vacuum

\section{INTRODUCTION}

Melanins are umber and black pigments, wide-spread in the vegetable, animal and microbial kingdoms of Nature. They are contained in hairs, eyes, skin, in many internals, but mainly localize in the integumentary parts of organism. Dark seed, berries, patterns on the petals of flowers and leaves of many plants, tan of man, skin of negroes, whimsical coloration on hide of many animals (zebra, leopard, cheetah, jaguar etc.) the dark color is obligated to melanins. Generally it is known these pigments execute in living organisms protective functions against the action of UV-radiation, ionizing radiation, high and low temperatures.

However melanins possess a number of signs and features that does their unique substance and object of the most intent attention of researcher, scientists, philosophers, representatives of spiritual sciences, beginning from Aristotle and concluding our contemporaries. In the previous article in detail achievement in area of study of melanins during a more than 3000-years-old period are described and the row of inexplicable phenomena (facts, questions for which modern science can't give answers) is parallel presented [1]. The main from them are questions about biological functions and role dark pigment in Nature. Thus scientists acknowledge that the true role of melanins in Nature is not found out. Protective and adaptive functions are the top of iceberg and essence of melanin pigments lies much deeper and for its comprehension bringing more deep scientific and philosophical approaches are needed $[2,3]$.

At the same time in the enormous array of scientific information there are numerous facts and information which straight or indirectly specify on active participation melanins in energy-and- informative exchange in Nature. Foremost it is process of ante-biological evolution and origin of life on Earth, forming, development and further evolution of living organisms, mechanisms of thought, memory and consciousness.

In our opinion a structure of melanin is two-tier: material and field simultaneously. All previous research touch exceptionally the material level. There are grounds to suppose that all inexplicable facts are related to the field structure of melanins.

In 1995 on International Congress in Prague Italian professor P.A. Nicolaus, the highest authority in area of research of melanin pigments, put a question: what are melanins, garbage or biological jewels. In this connection consideration of foregoing facts and information from positions of the newest scientific theories - the Theory of Torsion Fields (TTF) and the Theory of Physical Vacuum (TPV) - appears actual and timely and will promote even to the partial removal of the blank in knowledge about melanins.

\section{Classification, Structure AND Properties of Melanin Pigments}

Term "melanin" originates from Greek word $\mu \varepsilon \lambda \alpha \zeta$, i.e. black. However there is an enough vast group of hasel and yellow pigments which are biologically relational to melanins. Therefore R.A. Nicolaus offered light pigments to name pheomelanins (from pheos - yellow, red) and umber and black pigments - eumelanins (from eu - good) [4]. Both these terms concern to the pigment of animals. In respect of dark matter of plants, both higher and lover, they join in the group of allomelanins, in the number of which melanins of 
higher plants phytomelanins are selected. On a Fig. 1 the structural formulas of most studied melanins are presented.

a)

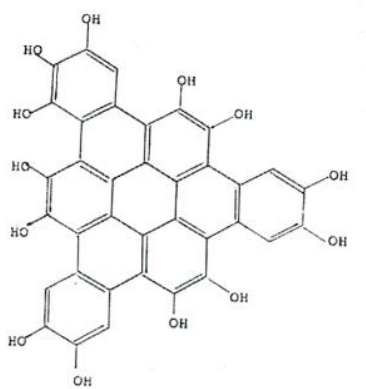

c)

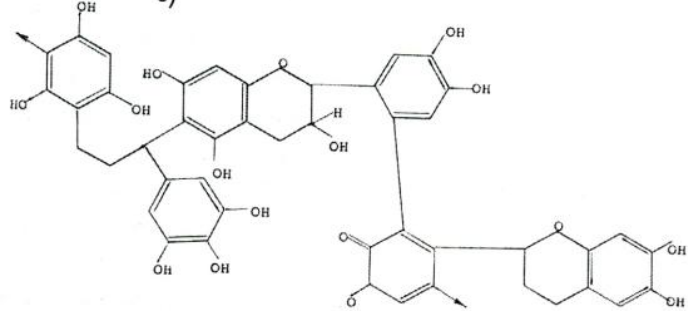

Fig. 1 Supposed structural formulas of some melanins: a) Ustilago maydis-melanini; b)

Aspergillus niger-melanin; c) Vitis vinifera L.melanin

Selected that or by other method melanin is in dried up kind is black or umber powder with metallic brilliance. It is an amorfous matter the disorderly orientation of monomeric units. Melanins are insoluble in most mineral and organic solvents. Universal and the almost unique solvents are bases $\mathrm{NaOH}$ or $\mathrm{KOH}(0,5-1,0 \mathrm{M})$. On a Fig. 2 preparations of phytomelanins (got by author of this article) are presented. '

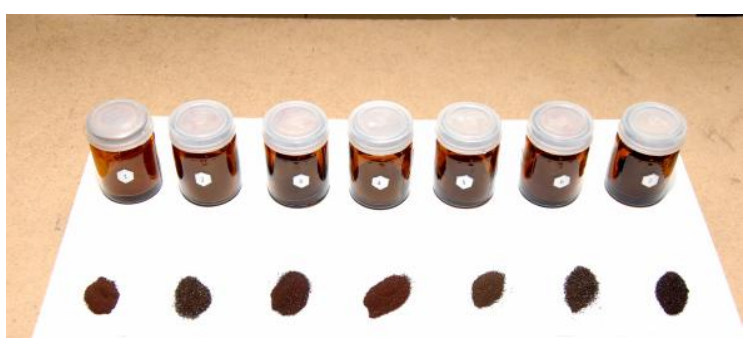

Fig. 2 External appearance of phytomelanins

All melanins possess paramagnetic properties which first have been found by Kommoner in 1954 by method of electronic spin resonance (ESR). Paramagnetic properties testify to presence in the matter of stable free radicals.
Melanins show ESR-absorption as a slightly asymmetric singlet with g-factor within the limit of $2,0030-2,0040$. The closeness of melanin g-factor to the g-factor of stable unpaired electrons 2,0023 testifies to the closeness of their properties. Concentration of paramagnetic centers in melanins is within the limits $10^{17}-10^{21} \mathrm{spin} / \mathrm{gram}$. A presence of signal ESR is so characteristically for melanins that absence of this signal in a black pigment unreservedly calls a doubt in a belonging of this pigment to the class of melanins. On a Fig. 3 the ESR-signal of grapes melanin is presented.

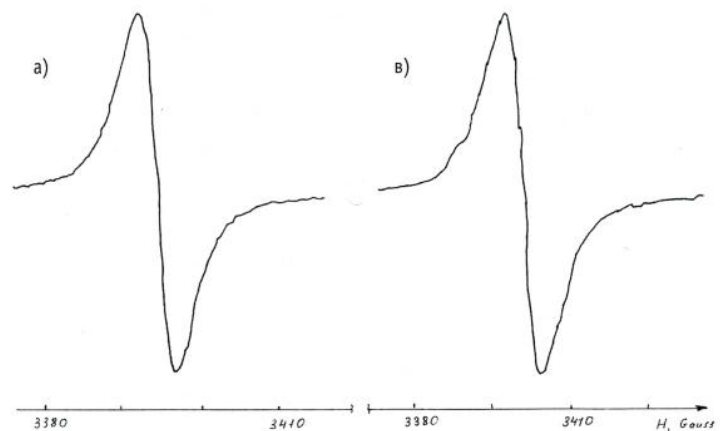

Fig. 3 ESR-signal of grape melanin: a) fresh pigment $1980^{\text {th }}$; b) the same pigment after 24 years in $2004^{\text {th }}$

\section{PECULIARITIES OF MELANINS}

In unanimous opinion of numerous researchers melanins occupy the quite special position among other biogenetic polymers on Earth. Many signs testify to it: physical and chemical properties; a place in the evolution of ante-biological and then biological structures; method of synthesis; wide distribution in Nature; "slipness away" from researchers, i.e. research inaccessibility to study by known science methods; many useful properties (antioxidantive, radio protective, tumor protective, antistressive etc.). Scientifical and technical literature have at one's disposal the numerous examples of the indicated properties. We will bring only some of them.

As marked already, all melanins possessed a high paramagnetism. This property is characteristic for the living state of matter. Melanins are unique they save the ESR-signal both in vivo and in vitro during very long time and does not lose it even at every hard chemical treatments. On this unique property of melanins a prominent scientist A. SzentGiorgy specified [5]: "The more intensive life, the higher of metabolism in one or another tissue and the more intensive ESR-signal from this tissue. After death an organism paramagnetic properties are lost and diamagnetic receptivity increases. ESR-signal is presumably the signal of Life. Only very not much stable matters demonstrate this signal, foremost melanins. 
Unlike many other pigments melanins were synthesized as very as period of ante-biological evolution, synthesized now and inherent all now living organisms on Earth.

Melaninogenesis is a not permanent process. It is a changeable radical process, strongly oxygen-sensitive, to the peroxides, $\mathrm{pH}$, to the concentration of reagents etc. In Nature are not two identical melanins even if to come from the same precursor. How synthesis melanin in vivo? It remains a mystery.

There is a hypothesis the neuromelanin (melanin of the brain) is a self-organizing polymer. The internal self-organizing straight correlates with the embryonic ripening. Therefore a supposition was done that unique mental endowment ("mind") of every individual has a direct relation to his unique neuromelanin structure.

The concentration melanin in the brain increases as far as advancement on phylogenetic stair arriving at a peak for man.

Melanin is the most universal pigment in living organisms beginning from primitive mushrooms and concluding primates [6, 7]. At vertebral melanin is contained not only in a skin but also in many other vital organs: nervous system, hypophysis, hypothalamus, epiphysis, thyroid, heart, liver, buds, blood, muscles, gonady etc. In the period of embryonic development melanin is present on every stage, beginning from oocytes. Then the pigment concentrates in great quantity in a nervous crest, from which take beginning nervous system, neuroendocrine system, skin etc. Thus it is possible to consider that melanin in living organisms executes some important function exceptionally on the basis of this everywhereness.

Extraordinarily high chemical stability of melanins allows them to remain "chemical fossils". Melanocytes had been found in an unchanging kind in the remains of ichthyosaurus, decapods shrine age of which was calculated in 150 million years $[4,11$, 12]. In more late time melanins in abundance had been found in human mummies [13].

Melanin is an extraordinarily steady molecule with high-resistivity to the action of acids, bases, organic and mineral solvents and also to the modern technique of analysis. It does him little accessible for study, by a "black box" (the term is entered by the American scientist from Institute for Study of Consciousness) [14].

All of it did melanin one of interesting and attractive object of scientific research from Aristotle to our days. The most essential results concern to chemistry, biology, pharmacology, enzymology of melanin pigments. Laboratories and research centers are on all continents in many countries of the world. The most complete knowledge about melanin are got Italian professor R.A. Nicolaus.
The row of researchers got major practical results in application of melanin for treatment of such diseases as AIDS [15], Alzheimer's, Parkinson's, other degenerative disorders of this type [16]. On the melanin basis numerous screen are created for protecting from harmful electromagnetic radiation [17].

A large deposit was done by soviet scientists in area of research of microbial melanins [18], phytomelanins [19], grape melanins [20, 21]. On the basis of grape melanin the methods of stabilizing of wines are developed $[22,23]$. After the failure at the Chernobylskaya Atomic Station grape melanin was used for cleaning of liquids from radioactive contaminations [24].

But in spite of so deep and comprehensive researches in area of knowledge about melanin still there is a number of inexplicable facts. The numerous scientific articles, as a rule, end with questions which have not any answer in modern science. Main from them are following.

What is the biological role of these pigments?

What is the veritable function of melanins?

What is the function of melanins in internal organs of man and monkey?

Mechanisms of protective function, melanogenesis to the end are not found. Unbelievable stability of melanins in far for living state terms of temperature, $\gamma$-radiation, time is not explained.

Therefore R.A. Nicolaus marked that at research of melanins it is necessary to attract new approaches, not only biological, chemical, physical, but also philosophical [25].

In our opinion it is possible to search answers for the put questions only by researching of energy-and-informative properties of these pigments. Possessing such exceptional property as a paramagnetism and by such high quantity of the unpaired electrons melanins participate in those energy-and informative processes which take place in Nature. Presently only two physical theories detailed on a strict scientific basis describe energyand-informative processes. It is a Theory of Physical Vacuum (TPV) and a Theory of Torsion Fields (TTF) [26, 27, 28, 29]. In opinion of many scientists these theories make the new scientific paradigm of XXI century. Extraordinary in these theories is that they entered in Physics a new kind of interactions, fifth force, long-distance Torsion Field or spin field.

The torsion field possesses many unusual properties. But the most important is that the source of torsion field is spin or quantum analog of angular rotation moment. Exactly this fact supposes a presence the torsion field in melanins and, as result, unusual properties and display of them in the material world. Further we bring analysis some scientific facts in the light of these new theories and present got results. However at the beginning we 
short consider some position of new theories which have a direct relation to understanding of such difficult structure as melanin pigment.

\section{BASIC POSITIONS OF THEORY OF PHYSICAL VACUUM AND THEORY OF TORSION FIELDS}

TPV proves that surrounding reality is a seven levels hierarchy of different forms of matter existence (Fig. 4). One of these levels is the primary torsion field or informative field, or field of consciousness.

\begin{tabular}{ll} 
VII & Absolute Nothing \\
\hline VI & Primary Torsion Field \\
\hline V & Physical Vacuum \\
\hline IV & Plasma \\
\hline III & Gas \\
\hline II & Liquid \\
\hline I & Solid \\
\hline
\end{tabular}

Fig. 4 Seven levels of reality

The source of the torsion field is a classic spin or quantum analog of angular moment of rotation. In macrocosm the source of the torsion fields are rotating masses.

In the surroundings of spinning object the torsion field is distributed as two spatial cones with right and left polarization. Thus the torsion field has axial symmetry unlike the electromagnetic and gravitational fields having central symmetry (Fig.5).

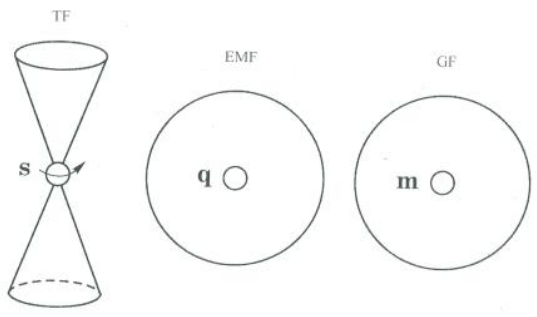

Fig. 5 Structures of torsion, electromagnetic and gravity fields

The torsion field is quantum whirls of space-time. It carries information without a transfer of energy. Therefore it yet named the informative field or by the field of consciousness.

The torsion fields are right and left depending on direction of spin. They possess highest penetrable ability. Speed of distribution of the torsion fields is equal endlessness.

Unlike electromagnetism the law of similarity operates in the torsion fields: of the some name charges (parallel spins) attract and the opposite charges (anti parallel spins) push off.

The torsion fields possess "memory". This memory is an exactly orientation of spins. An object sensible to the action of the torsion field is a spin system. The system of spins takes advantage in regard to a size effect as compared to the individual spin of particle. Such system is the original strengthener for small effects of every separate spin. All matters of Nature have the own individual torsion field. Superposition of torsion fields created by the atomic and nuclear spins of every molecule determinates the torsion field in the space around this molecule. The spatial-frequency structure of the torsion fields is determined chemical compositions of molecules and their spatial configurations. Complication and stability of spatial-frequency structure of the torsion fields is major condition for the carriers of information.

In the light of these positions pigment melanin with its high level paramagnetism and unpaired spinning electrons appears as certain special torsion matrix inherent in living matter. The torsion field in space around the melanin molecule is very complicated spatial-frequency structure.

Coming from extraordinary stability of melanins in time it is possible to assert that this structure of torsion fields is also steady in time. It means that melanins can be examined not only as transmitters but also as receivers of information. Presumably melanin is able to receive, accumulate, keep and carry information from the higher levels of reality to the levels of matter. These processes are carried out due to stable lone electrons which are deeply included in the melanin matrix yet at its birth and remain there forever. These electrons are responsible for the paramagnetism and ESR-signal. Probably Nature thus saves and passes "Signal of Life" both in vivo and in vitro in the kingdom of living matter.

\section{SCIENTIFIC FACTS TESTIFYING TO THE TORSION APPROACHES}

5.1. Melanins as organic catalysts on primitive Ears

American biophysicist M. Blois was the first who paid regard to melanins as on an organic matrix for processes of chemical evolution [30]. He not only in theory grounded the role of melanin pigments in the processes of synthesis antebiological structures but also experimentally confirmed on the example of-DOPA-melanin participating of free radicals in the processes of polymerization. Moreover in this work he indicated on the priority role of melanins among other important molecules: "It is always risky to consider at our modern understanding of chemical evolution that one biological molecule is more "primitive" 
than other. And all the same it is not eliminated that on a question: "What polymer did arise up before, protein or DNA?" we will get an answer: "Melanin".

The conclusion of the American biophysicist was confirmed by Soviet researchers at the study of abiogenous synthesis of peptides in the modeled terms taking place on young Earth in the period of chemical evolution [31, 32].

In these works melanin was examined as a product of "deadlock" synthesis of proteins, enzymes, DNA and similar materials which could not be carried out by reason of too hard terms (UV-, $\gamma$-radiation, space rays etc.) on primitive Earth.

Then in the light of TTF melanin can be examined as a matrix in the torsion field of which there is memory about of materials the synthesis of which came to deadlock at one time (Fig. 6).

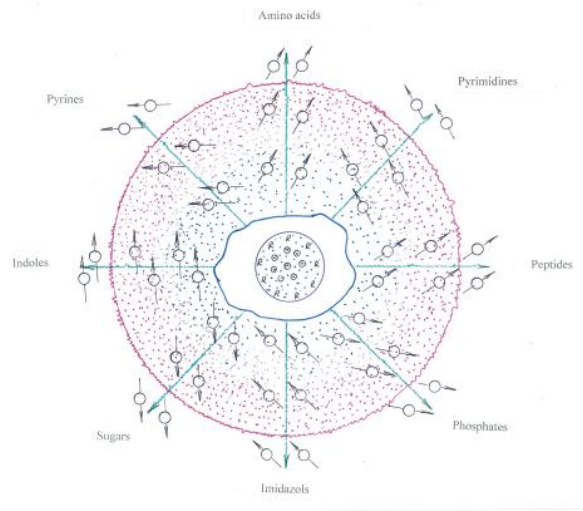

Fig. 6 Torsion field of melanin matrix

Due to this memory melanin can be catalyst in the processes of chemical evolution. How does it show its catalyst properties? Coming from the law of similarity it is possible to assert that the melanin matrix, operating through the torsion field, attracts and absorbs any not molecules but only those at which spatial and frequency descriptions are similar to its own. Such materials are amino acids, peptides, sugars, phosphates, purines, pyrimidines and other initial products for synthesis of biopolymers, because memory about them is reliable kept in torsion field of melanin matrix.

At the interaction of torsion fields matrix and precursors there are spin reconstruction at the matter level which execute the role of "trigger-hook" or catalyst which includes the synthesis of biopolymers. The appearing biopolymers are reliably depended by melanin matrix from harmful radiation.

Probably Nature in search of exit from a deadlock on the certain stage of chemical evolution created Melanin. It decided by melanin two tasks simultaneously. At first, it reliably hid and saved information-plan of future biopolymers. Secondly, it created favourable terms for creation of living matter. More information on these questions is contained in the article [33].

\subsection{Dominant role of melanins in origin of life}

In obedience to new physical theories origin of life is a key event in the chain of successive transformations of the field form of matter in material form. From these positions the living state can be described as the special correlation between the field form and the material form of matter at fully certain level of reality. Torsion field around material part is the informative constituent of living organism or his biological field (biofield). Thus there is an appropriate question: what must be this correlation in numerical expression? Today it is scarcely possible to give an exact answer on it. However much approximate calculations on the basis of informaciology appear possible.

From informaciology [34] it is known that all space of Universe is in $99,999999 \%$ from Information and only in $0,000001 \%$ from matter. On that ground it is possible to consider that the main sign of living matter is its highest informative capacity at minimum maintenance of matter. From all known materials having in living organisms only melanins posses a similar sign because they contain in their mass unpaired electrons which create additional field component.

Approximate calculation show that even if to consider that the carriers of information in melanins are only the unpaired electrons then informative and material parts are 99,9999995\% and $0,0000005 \%$ accordingly. Thus from a point of informative capacity melanin is a key molecule on "turning" stage of evolution when matter and laws of material world begin to play a noticeably role. More complete information on these questions is contained in the article [35].

\subsection{Embryogenesis and mechanisms of melanin action}

Embryonic development (embryogenesis) is an aggregate of morphological and physical processes as a result of which the complicated highdifferentiated organism from one primary cell zigota. Division, growth and differentiation of oosperm and formation in the end of human being it is one of the surprising and intriguing phenomena of biology. Scientists acknowledge there is a number of questions which are distant from decision in modern biology [36]. There are only a few examples here. What is a reason of differentiation of cells?

How is managed embryogenesis?

Why cells of the same organism and having the same genetic material does differ such wide variety typical for human being? As far as development of embryology and accumulation of experimental facts gradually understanding appeared that forming of living organism can't be carried out only under the management of material factor - 
gene. Loading on a gene is too great. Therefore assumption about possible participation of the field factor was done. In behalf of the field factor that manages by embryonic development unique experiments of German embryologist H. Drish testified [37]. A decision step in development of the field conception of embryogenesis did Soviet biologist A. Gurvich. He entered concept of the biological (morphogenetic, embryonic) field [38, 39]. Not considering physical nature of the morphogenetic field he formulated its properties fully certainly: long-distance action, attraction and pushing away, symmetric sharp character. It is necessary to underline that all this properties of the morphogenetic field full coincide with long-distance torsion field.

In the theory of embryogenesis the special role is taken epithelial cells $[39,40,41]$. It is known the feature of epithelial cells is very high quantity in them of melanin [14]. In addition in embryology it is well known that on the early stages of embryo development melanocytes are intensively formed. They form large accumulation as a black fat fold. Later from this fold vitally important structure form. It is CNS, spinal cord, thyroid, endocrine system, adrenal glands, skin etc. Thus all vitally important structures originate from melanocytes. We worked out model of embryogenesis on the basis of melanin action mechanisms and presented a row evidences in its behalf [42].

\subsection{Melanins, Consciousness, Mind}

At the beginning of the last century N. Bor put an idea that future physics must will be included in itself consciousness as a physical reality. More than 50 years the idea of $\mathrm{N}$. Bor did not find real scientific ground in physics. And only TTF allowed on a strict scientific basis to interpret concept of consciousness, mind, mechanisms of thought and memory[27]: "Consciousness and Thought, and in limit World Mind are presented in Physical Vacuum not abstractly, but through concrete essence Torsion Field... Consciousness as a functional structure includes in itself a spin biological computer - brain and its external part - torsion calculable machine which is the Physical Vacuum polarized on spins in space around the brain".

In TTF it is assumed the external torsion field through the state of some materials of neurons can influence on the state of neurons, processes associate memory, imaginative thought etc. However many author underline that it is not clear what concrete chemical compounds of living organism would participate in the transmission of torsion influences.

On the other in $80^{\text {th }}$ of the last century a hypothesis was advanced that neuromelanin functions as the major organizing molecule in living systems [14]. Authors supposed that melanin was a "reasonable" molecule or transmitter Reason in living organisms. In behalf of this hypothesis they brought a row over of surprising facts. However in subsequent years this hypothesis did not get development. Presently it remains one of the most enigmatic and "narrow" place in vast researches of melanin pigments.

In our view reason of such position consists in ignoring of informative approaches. Interaction Melanin - Mind is beyond an only material problems on two reason. At first, category Mind is more informative than material concept. Secondly, melanin of itself is two levels substance, including organic matrix and informative (torsion) constituent as spinning electrons around. Therefore of research of this hypothesis it is necessary to take into account not only material level but also level of information, i.e. torsion field.

We analyzed this hypothesis from positions of TTF and TPV and got irrefutable proofs in its behalf. Torsion approaches and hypothesis of Barr appeared not only compatible but also mutually complementary each other [46, 47]. We give only two concrete examples.

For example, neuromelanin in this hypothesis appears as trigger, switching and shutting cascades of biochemical reactions. A key factor here is covalent bonds. Unlike ionic bonds, metallic bonds and forces of Van-der-Waals covalent bond is formed by two spinning electrons. Therefore it is more labile and sensitive to the action of torsion fields. At the hit of the external torsion field on neuromelanin there are changes foremost in spins of covalent bonds. As a result of these changes is intramolecular reconstruction of proteins, DNA, amino acid, purines etc. So neuromelanin is possible to switch and shut cascades of biochemical reactions.

Another example is here. As it was marked before new spin structures in space around the brain arose. They are reflected as images in our consciousness (Fig.7).

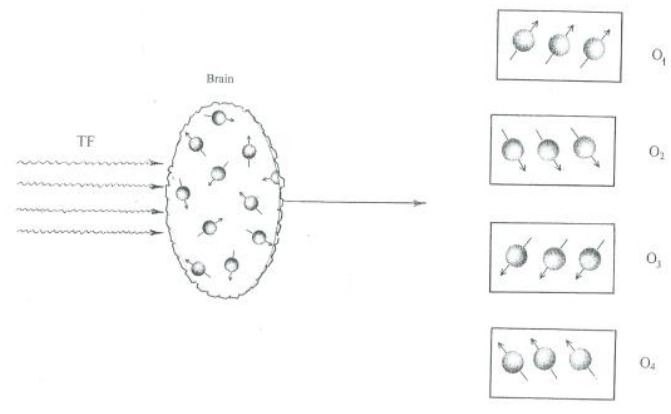


Fig. 7 Interaction neuromelanin with external torsion field. $\mathrm{O}_{1}, \mathrm{O}_{2}, \mathrm{O}_{3}, \mathrm{O}_{4}$ - reflected images in consciousness

According to TTF these images make our consciousness, provide thought and memory and also can be perceived by other brain. They are also responsible for psychophysical interactions. Then there are all grounds to suppose that a violation of neuromelanin metabolism can result in different diseases. In reality this fact is observed. It is showed that a number of degenerative diseases and psychical disorders are related to exhaustion of neurons and loss by them of melanin $[16,48]$.

\section{RESULTS OF OWN RESEARCHES}

During a row years the author researches into melanins of higher plants, phytomelanins. As a result technologies of select are developed and preparations of different plants (grape, chestnut, buck-wheat, sunflower, sea-buckthorn, tea, cacao) are selected. A principle possibility of the use of phytomelanins is proved for creation radio protective preparations of medical and prophylactic action, for decontamination of radioactive liquids, for lighting and stabilization of juices and wines. These technologies are executed at level of inventions and fastened Copyright Certificates the USSR [22, 23, 24]. In the subsequent years with the Slovakian colleagues the bioactive fraction of vegetable melanin was selected and investigated [49]. On its basis the product with the commercial name "Matuzalem" was development. This product has medical and prophylactic properties. During the prolong work also some inexplicable phenomena and paradoxes appeared.

For example preparations selected by different authors show different biological activity. They in living organisms work variously in spite of identical physical and chemical indexes. In the environment of experimenters an opinion took root even: "one man - one melanin".

Looking after the medical and prophylactic operating Matuzalem on organism of man allows to draw conclusion that it works at informative level. For one man it treats one, at other - other.

It was found the phytomelanins were able to neutralize (to remove) geo-pathogenic areas [51]. So standing grape melanin on a pathogenic area during an hour removed in complete pathogenic sign. Analogical action was rendered by product Matuzalem.

Within in the frame of existent theories explaining these facts is impossible but by torsion approaches they can be explained enough simply. Possessing a complete spin structure melanin perceives external torsion fields including torsion field (bio field) of one or another operator. These imprints (tracks) are memorized by torsion field of preparations. In future they will influence on preparations properties.

By method of Gas-Discharge-Visualization [50] we also investigated energy-and-informative properties of melanins and Matuzalem. Energy-and-

informative potentials appeared very high and comparable with the most active and useful to man products such as maternal milk, colostrums, honey, etc.(Fig.8).

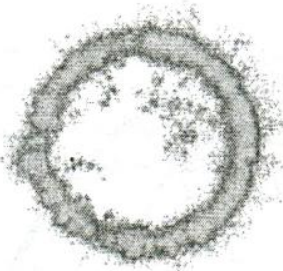

1

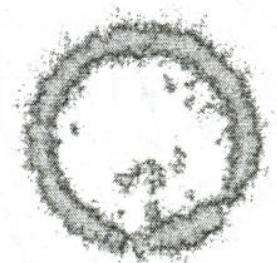

3

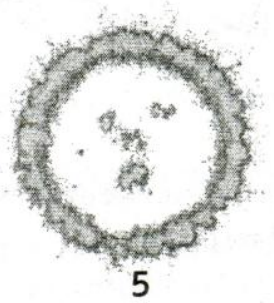

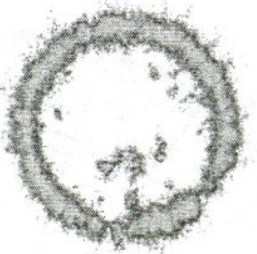

2

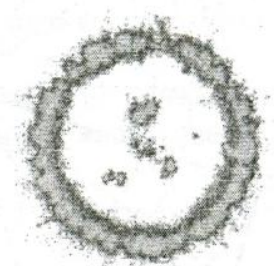

4

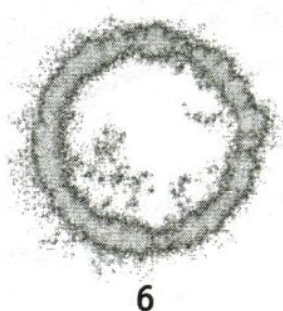

Fig. 8 GDV-grams of phytomelanins: V. vinifera L. (1), Thea L. (2), C. sativa L. (3), T. cacao L. (4), Heliantus annus L. (5), V. vinifera L. soluble form

(6)

Thus distribution of these potentials on the systems and organs considerably excels value for healthy state(Fig.9).
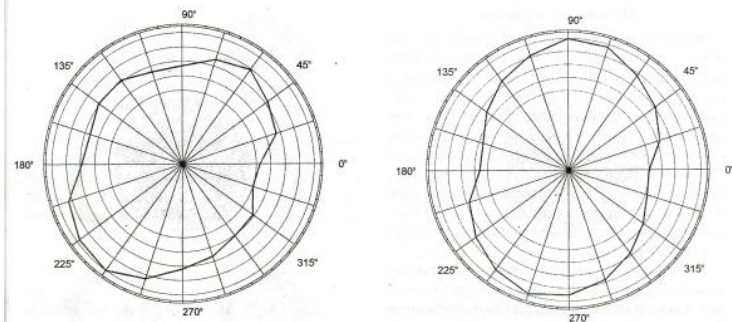

Fig. 9 Energy-and-informative potential V.vinifera L.-melanin (left) and product Matuzalem (right)

Therefore it is not surprising that Matuzalem itself finds "defects" in organism. In respect of geo pathogenic areas their veritable nature and mechanisms of action are not exposed. According to the set opinion in science about Earth 
there are different types of geo genic energies as a result of its rotation in cosmic space [52]. Dominative value is taken by torsion "energy". Possibility formation of electronic states at atoms in minerals of mountain breeds is not eliminated also [53]. On the other hand most people possess the right torsion field. And only one man on million is with left torsion field [29]. Therefore it is natural to suppose that geo-pathogenic areas are sources of the left torsion field which renders the ruinous operating on living organism. Fully probably that melanins as sources and transmitters of the right torsion field are able to change spin polarization of physical vacuum in geo-pathogenic areas on opposite right which is favourable to living organism.

\section{CONCLUSION}

Pigment melanin is a different material substance including two levels of organization: material and field. Material level is complicated three-dimensional irregular organic matrix built from atoms $\mathrm{C}, \mathrm{O}, \mathrm{H}, \mathrm{N}, \mathrm{S}$. Field level is the torsion field around organic matrix the source of which is the system of unpaired spinning electrons belonging exceptionally to melanins. Due to steady system of spins melanin matrix is transmitter of the most complicated structure of torsion field in the wide range of spatial, frequency and temporal characteristics.

Melanin matrix is able to perceive, write down and keep information at the level of its spins and to participate in energy informative processes at all levels of development living matter: from chemical evolution to function of brain and consciousness.

Pigment melanin is an instrument of Nature for storage and distributing Information in the world of living matter. Properties of spin structures and effects of Torsion Fields underlay a basis for action mechanisms of melanin.

On today's TTF and TPV are new and did not get wide distribution in science yet. Therefore pigment melanin continues to remain by surprising, most enigmatic and mysterious biological polymer of Nature.

Melanin possesses a number of unique priceless properties. Therefore it is a gift of Nature to Man, in truth his Diamonds.

\section{REFERENCES}

[1] L. Venger. Enigmas and mysteries of melanin pigments, Eniologiya, 4(16), 2004, 56-63.

[2] B.J.R. Nicolaus, R.A. Nicolaus. Progress in Physical Chemistry of Melanins. Scientific Communication

I, http//www.tightrope.it//nicolaus.

[3] B.J.R. Nicolaus, R.A.Nicolaus. Melanins, garbage or biological jevel, Oral
Communication presented of the VIII Meeting of the European Society for Pigment Cell Research. Sept. 23 - 26, 1998, Prague, Czech Respublic. http//www.tightrope it//nicolaus.

[4] R.A. Nicolaus. Melanins (Paris: Hermann, 1968).

[5] A. Scent-Georgy. Introduce in molecular biology (M.: Nauka, 19640.

[6] [6] T.Sarna. Interaction of melanin with oxygen, Archivas of Biochemistry and Biophysics, 250(1), 1980, 140-148.

[7] I. Menon, M. Haberman. Mechanism of action of melanins, British Journal of Dermatology, 97, 1977, 109-112.

[8] H.Wasserman. Melanin: cutaneous and extracutaneous, Ethnic Pigmentation, (Amsterdam: Excerpta Medica, 1974) 133150.

[9] M. Altschule, Z. Hegedus. The importance of studying visceral melanins, Clinical Pharm. and Therap., 19(2), 1976, 123-134.

[10] N.le Douarian. Migration and differention of neural crest cells, Current Topics in Developmental Biology, 16, 1980, 31-35.

[11] F. Daniels, P. Post, B. Jonson, Theories of the role of pigment in the evolution of human races, in Pigmentation: Its Genesis and Biologic Control, (New York: ApplitonCentury-Crolts, 1972) 14-21.

[12] H.Wasserman. An holistic concept of ethnic pigmentation, Ethnic Pigmentation, (Amsterdam: Excerpta Medica, 1974) 264266.

[13] P. Post, F. Daniels. Histological and histochemical examination of American Indian scalps, mummies and a shrunken head, American Journal of Physical Anthropology, 30, 1969, 269-293.

[14] F.E. Barr, J.S. Saloma, M.J. Buchele. Melanin: The Organising Molecule, Medical Hypothesis, 11, 1983, 1-140.

[15] D.S. Montefiori. Method of inhibiting replication of HIV with woter-soluble melanins, US Patent. 5, 057, 325, Oct. 15, 1991.

[16] D.L. Berliner, R.L. Erwin, D.R. Megel. Theraputic uses of melanin, US Patent.5, 817, 631, Oct. 6, 1998.

[17] J.M. Gallas. Medium incorporating melanin as an absorbing pigment against electromagnetic radiation, US Patent. 5, 112, 883, May 12, 1992.

[18] S. Lyach, R. Ruban, Nicrobial Melanins (M.: Nauka, 1972).

[19] N. Bidzilia. Free radicals in radiationexposed plants and seed (K.: Naukova dumka, 1972). 
[20] A. Bogatskiy, Yu. Zherebin, S. Makan. Melanins in wines, Wine making and viticulture of the USSR, 9, 1979, 52-54.

[21] Yu. Zherebin, S. Makan. Flavanolic nature of melanins in wine. Lecture AN Ukrainian SSR, 7, 1981, 46-49.

[22] R.Sendega, L. Venger, L. Baklanova. Method of receipt of enomelanin, Copyright Certificate the USSR, № 1345606, June 15, 1987

[23] R. Sendega, L. Venger, L. Baklanova. Method of enomelanin regeneration, Copyright Certificate the USSR, № 1336556, May 8, 1987.

[24] N. Bidzilya, L. Venger, L. Timofeeva. Method decontamination of liquids, Copyright Certificate the USSR, № 1522714, July 15, 1989.

[25] R. Nicolaus, Stydies of Melanin (htt://www.tightrope.it//nicolaus).

[26] G. Shipov, Theory of Physical Vacuum (M.: Nauka, 1997).

[27] A. Akimov, Heuristic discussion problem search of new long-range actions. EGSconceptions, in A. Akimov (Ed.), Consciousness and Physical World. Collection articles, 1 (M.: "Yachtsmen", 1995) 28-43.

[28] A. Akimov, G. Shipov. Torsion fields and their experimental displays, Consciousness and Physical Reality, 1(3), 1996, 28-43.

[29] A. Akimov, Look of physics and thechnologies at the beginning XXI century (Novosibirsk: Sibprint, 2003).

[30] M. Blois. Disorderly polymers as matrix for chemical evolution, in Origing of prebiological systems (M.: Mir, 1966) 27-47.

[31] T. Pavlovskaya, T. Telegina. Pre-biological synthesis of biochemically important compounds, in Abiogenes and initial stages of life (M.: Nauka, 1968) 41-48.

[32] T. Telegina, T. Pavlovskaya. Catalysts of melanin-melanoidin type are in abiogenes of peptids, News AN the USSR. Series biological, 1, 1988, 112-117.

[33] L. Venger. Role of melanins in chemical evolution, Eniologiya, 3(23), 2006, 21-29.

[34] I. Yuzvishin, Basis of informaciology (M.: Higher school, 2000).

[35] L. Venger. Dominant role of melanins in life and evolution of living organisms, Eniologiya, 4(24), 2006,34-49.

[36] N. Green, G. Stout, D. Taylor, Biology (M.: Mir, 1990)

[37] L. Liozner, Basic problems of study about regeneration (M.: Nauka, 1975)

[38] A. Gurvich, Theory of the biological field (M.: Sovetskaya nauka, 1944).
[39] A. Gurvich, Select labours (N.: Medicine, 1977).

[40] B. Belincev, Selforganization in development of embrio, Nature, 2, 1989, 84-89.

[41] B. Belincev, Physical bases of biological forming (M.: Nauka, 1991).

[42] L. Venger. Embryogenes and mechanisms of melanin action, Eniologiya, 3-4(27-28), 2007, 16-26.

[43] A. Akimov, V. Bingi, About Physics and Psychophysics, in A. Akimov (Edit.), Consciousness and Physical World. Collection articles, 1 (M.: "Yachtsmen", 1995) 104-125.

[44] A. Akimov, G. Shipov, Consciousness, Physics of Torsion Fields and torsion Technologies, Consciousness and Physica Reality, 1-2(1), 1996, 66-72.

[45] A. Akimov, V. Bingi, Computers, Brain and Universe, in A. Akimom (Edit.), Consciousness and Physical World.Collection articles, 1 (M.: "Yachtsmen", 1995) 126-136.

[46] L. Venger. Physical models of brain and neuromelanin, Eniologiya, 3-4 (27-28), 2007, 11-122.

[47] L. Venger. Melanin as transmitter of Mind in living organisms (in print).

[48] D. Berliner, R. Erwin, D. Megel, Theraputic uses of melanin, US Patent, 5, 518, 631. Oct. 6, 1998

[49] J. Kerestesh, J. Kerestesh, L. Venger, Biologically active fraction of vegetable melanin, process for its productionnand its use, US Patent, 6,576,268. Jun 10, 2003.

[50] L. Venger, J. Kerestesh. Energyinformative properties of product "Matuzalem", Eniologiya, 2(14), 2004, 59-63.

[51] G. Pilipenko, H. Charskaya, O. Zurkan, Objectivization and neutralization of geoactive structures of local level, in Materials of $V$ International Congress "Eniologiya XXI th century". 16-19 sept. 2004 (Odessa: ENIO, 20040 178-180.

[52] A. Drozdovskaya, Hystory and further fate of humanity in influences of kosmic and geogenic fields, Eniologiya, 3(13), 2004, 2329.

[53] K. Balandin. Practical work on biolocation, Eniologiya, 1(11), 2003, 62-68.

Mail your Manuscript to ijera.editor@gmail.com 